\title{
Dynamical properties of a new six-term chaotic system
}

\author{
Sen $\operatorname{Qin}^{1, a}$ \\ ${ }^{1}$ School of Science, Hangzhou Dianzi University, Hangzhou, Zhejiang, 310018, China \\ aquinsen0425@hdu.edu.cn
}

Keywords: Chaotic system; Butterfly-shaped; Dynamical properties; Bifurcation diagram.

\begin{abstract}
A new butterfly-shaped three-dimensional chaotic system with six terms is proposed, which includes four parameters to control its chaotic dynamics. Some dynamic behaviors, such as symmetry, equilibrium points, and Lyapunov exponent spectra, are theoretically and numerically discussed. The bifurcation diagrams of the system with respect to two parameters are analyzed. The new chaotic system will provide a new meaningful template for developing the useful chaos and controlling chaos.
\end{abstract}

\section{Introduction}

The ability to understand practical impact of chaos is of utmost importance to real-world dynamical systems in many fields of science and technology, and has the potential to explore new effective methods of controlling inherent chaotic phenomena. The pioneering work of Lorenz [1] was exactly discovered the unpredictability of the weather patterns, and given a relatively simple three-dimensional autonomous chaotic system with quadratic terms, which is now known as the Lorenz system. Many researchers have since then paid strikingly attention to the studies of chaos. A lot of famous chaotic systems, such as Rössler system [2], Genesio and Tesi system [3], Chen system [4], Lü system [5], have been proposed over the past few decades. These results enable us to extend topological structure of chaotical phenomenon in a very natural way to the dynamic case.

In recent years, many studies of controlling and employing chaos are substituted for analyzing and understanding it as a new trend. Since the suggestion of two important feedback methods of controlling chaos: OGY method [6] by means of applying only very small perturbations to those unstable periodic orbits, and Pyragas method [7] using time-delayed controlling forces, many researchers have paid strikingly increasing attention to the studies of controlling performance for the extension of various feedback controlling methods. On the other hand, some non-feedback adaptive methods with external perturbations are suggested to produce drastic changes in the dynamics and to stabilize those periodic behavior of chaotic systems [8]. Generating chaos intentionally to play its advantages or changing chaotic property when it is harmful, have been succeeded in a variety of chaotic systems, including turbulent fluids and cardiac tissues [8]. Moreover, examples of the application of chaos range from biomedical engineering [9] to information security [10] and communications [11]. Therefore, it is necessary and meaningful to explore new chaotic systems and even control them, since chaotic phenomena widespread in real-world dynamical systems and chaos has a range of applications in many areas. It also allows us to track the evolution of chaos and inherent topological principle for designing new dynamical systems: avoid the harmful chaos and develop the useful chaos.

In the present paper, we are interesting in design and control of a new chaotic system with only six terms. It is a simple three-dimensional autonomous system that is easy to implement, and its first and third equations are similar to the Lü system but the second equation only contains a nonlinear term $-x(t) z(t)$. Some important dynamical properties of this new chaotic system, such as symmetry, equilibrium points, and Lyapunov exponent spectra, are discussed theoretically and numerically. And the bifurcation diagrams with respect to four parameters are also investigated. 


\section{A new butterfly-shaped chaotic model}

In this section, we establish a new butterfly-shaped chaotic model and give its phase diagrams with a group of initial states. Consider the following three-dimensional chaotic system

$$
\left\{\begin{array}{l}
\dot{x}(t)=-a x(t)+b y(t), \\
\dot{y}(t)=-x(t) z(t) \\
\dot{z}(t)=-c z(t)+x(t) y(t)-d .
\end{array}\right.
$$

where $a, b, c$, and $d$ are the system parameters. Obviously, in the second and third equations, each nonlinear term is formed by one cross product $-x(t) z(t)$ and $x(t) y(t)$, respectively. Similar to the third equation of Rössler system, we add a constant term $d$ to control the entire chaotic situation of the system. Fig. 1 shows phase diagrams of system (1) that four parameters are $(a, b, c, d)=(2,5,0.5,1)$ with initial states $(x(0), y(0), z(0))=(0.1,0.1,0.1)$. And the Poincaré maps projected in the $y-z, x-z$, and $x-y$ plane with $x=0, y=0$, and $z=0$, respectively, are showed in Fig. 2.
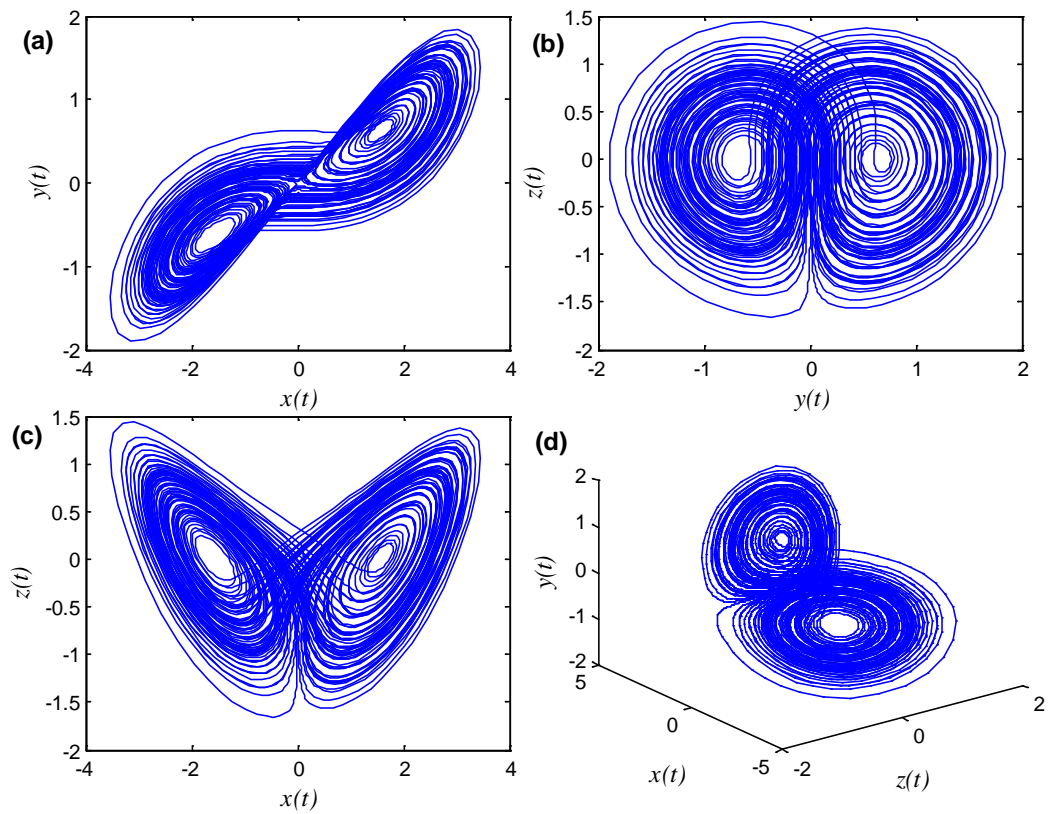

Fig 1. Phase diagrams of the new chaotic system. Here, $(a, b, c, d)=(2,5,0.5,1)$ and initial states $(x(0), y(0), z(0))=(0.1,0.1,0.1)$. (a) $x-y$, (b) $y-z$, (c) $x-z$, (d) $x-y-z$.
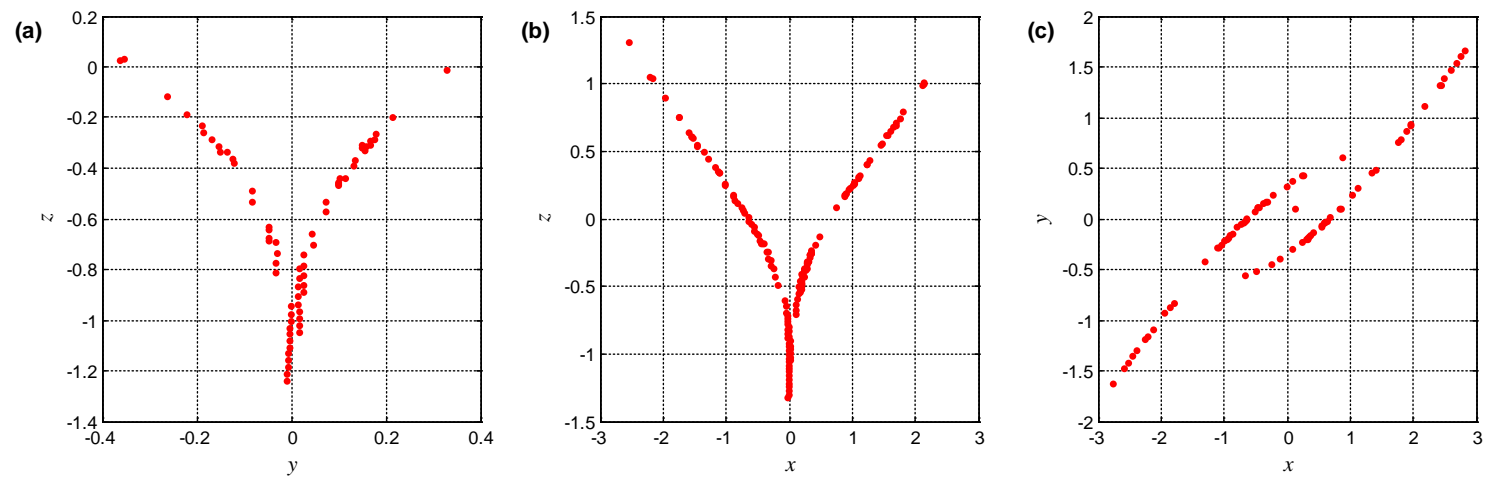

Fig 2. Poincaré map of the system (1) with $(a, b, c, d)=(2,5,0.5,1)$ and initial states

$$
(x(0), y(0), z(0))=(0.1,0.1,0.1) \text {. (a) } x=0 \text {, (b) } y=0 \text {, (c) } z=0 \text {. }
$$




\section{Dynamical properties of this new chaotic system}

Obviously, under the transformation of the variable $(x, y, z) \rightarrow(-x,-y, z)$, system (1) is invariant, which means this system is symmetric about the $z$-axis. Furthermore, it is invariant if introducing the transformation $(x, y, z, a, b, c, d) \rightarrow(x,-y,-z, a,-b,-c,-d)$.

Let $V=(\dot{x}, \dot{y}, \dot{z})^{T}$, we have

$$
\frac{1}{V} \frac{\mathrm{d} V}{\mathrm{~d} t}=\nabla V=\frac{\partial \dot{x}}{\partial x}+\frac{\partial \dot{y}}{\partial y}+\frac{\partial \dot{z}}{\partial z}=-(a+c) \text {. }
$$

Hence, for $a+c>0$ it is dissipative, which indicates the system can be convergent in an exponential rate $e^{-(a+c)}$. As $t \rightarrow \infty$, each volume that contains the orbits of the system converges into zero. And $a+c>0$ is required to fix the motion of the system on an attractor [12].

When considering the equilibria of system (1), the solutions of the following algebraic equations are calculated:

$$
\left\{\begin{array}{l}
-a x+b y=0 \\
-x z=0 \\
-c z+x y-d=0
\end{array}\right.
$$

When $\frac{b d}{a}>0$, the system has two equilibria $E_{ \pm}=\left( \pm \sqrt{\frac{b d}{c}}, \pm \frac{a}{b} \sqrt{\frac{b d}{c}}, 0\right)$; when $\frac{b d}{a}<0$, the system has still a unique equilibrium $E_{0}=\left(0,0,-\frac{d}{c}\right)$. Then, we study some properties of this new system, such as symmetry, equilibrium points, and Lyapunov exponent spectra.

As we know, the Jacobian matrix of a dynamical system describes its behavior near a stationary point. For system (1) at any equilibrium point $\left(x_{0}, y_{0}, z_{0}\right)$, its Jacobian matrix is

$$
J=\left(\begin{array}{ccc}
-a & b & 0 \\
-z & 0 & -x \\
y & x & -c
\end{array}\right)
$$

Let $|\lambda I-J|=0$, we obtain the character polynomial of this system

$$
\lambda^{3}+(a+c) \lambda^{2}+\left(a c+x^{2}+b z\right) \lambda+\left(a x^{2}+b x y+b c z\right)=0 .
$$

For two equilibria $E_{ \pm}$, Eq. 4 can be simplified as

$$
\lambda^{3}+(a+c) \lambda^{2}+\left(a c+\frac{b d}{a}\right) \lambda+2 b d=0 .
$$

According to the Routh-Hurwitz criterion, all roots of Eq.5 have negative real parts if and only if

$$
a+c>0, a c+\frac{b d}{a}>0, b d>0,(a+c)\left(a c+\frac{b d}{a}\right)-2 b d>0 .
$$

Let

$$
\begin{aligned}
& A=(a+c)^{2}-3\left(a c+\frac{b d}{a}\right), \\
& B=(a+c)\left(a c+\frac{b d}{a}\right)-18 b d, \\
& C=\left(a c+\frac{b d}{a}\right)^{2}-6(a+c) b d, \\
& D=B^{2}-4 A C, \\
& Y_{1,2}=A(a+c)+1.5(-B \pm \sqrt{\Delta}),
\end{aligned}
$$

we can get three solutions of Eq. 5 as follows: 


$$
\left\{\begin{array}{l}
\lambda_{1}=\frac{1}{3}\left[-(a+c)-\left(\sqrt[3]{Y_{1}}+\sqrt[3]{Y_{2}}\right)\right] \\
\lambda_{2,3}=\frac{1}{3}\left[-(a+c)+\sin \frac{\pi}{6}\left(\sqrt[3]{Y_{1}}+\sqrt[3]{Y_{2}}\right) \pm \cos \frac{\pi}{6}\left(\sqrt[3]{Y_{1}}-\sqrt[3]{Y_{2}}\right) \cdot i\right]
\end{array}\right.
$$

When either of the following conditions is satisfied: (i) $a>\frac{1+\sqrt{3}}{2} c>0, b>0, d>0$; or (ii) $a>\frac{1+\sqrt{3}}{2} c>0, \quad b<0, d<0$, one can get $A(a+c)+1.5(-B)=\left[a^{3}+c^{3}-1.5 a c(a+c)\right]+18 a b d$ $+4.5 b d(a-c) / a>0$. Hence, $Y_{1}>0$ and $\left|Y_{1}\right|>\left|Y_{2}\right|$, which indicates $\sqrt[3]{Y_{1}}+\sqrt[3]{Y_{2}}>0$. Furthermore, $\left|\lambda_{1}\right|>\left|\operatorname{Re}\left(\lambda_{2}\right)\right|$. Using Shil'nikov theorem [12], the vector fields of this system satisfy the conditions for generating chaos.

On the other hand, for the third equilibrium $E_{0}$, we have

$$
\lambda^{3}+(a+c) \lambda^{2}+\left(a c-\frac{b d}{c}\right) \lambda-b d=0 .
$$

Using similar analysis, we show that the chaos is generated when either of the following conditions is satisfied: $a+c>0, a c^{2}>b d,-b d>0$.

Then, in order to discover the average exponential rates of divergence or convergence of adjacent orbits in phase space of the system, its Lyapunov exponents are calculated by the Wolf algorithm with the original conditions $(a, b, c, d)=(2,5,1,5)$ and the number of iterations $t=100000$ : $\lambda_{1}=0.224600, \lambda_{2}=0.000014$, and $\lambda_{3}=-3.224616$. Although there will be a very slight gap if $t$ is different, the signs of three Lyapunov exponents are fixed as $(+, 0,-)$. When $(a, b, c, d)=(2,5,0.5,1)$ and $t=100000, \lambda_{1}=0.182833, \lambda_{2}=-0.000015$, and $\lambda_{3}=-2.682817$. Furthermore, its Lyapunov dimensions with two different sets of the four parameters are $D_{L 1}=2.069656$ and $D_{L 2}=2.068144$, respectively, which indicates that the attractor of the system has of fraction dimension.

Furthermore, Fig. 3(a), Fig. 4(a), Fig. 5(a), and Fig. 6(a) show that the bifurcation diagram of the system with respect to $a, b, c$ and $d$, respectively. These results show that the strange-shaped attractors with different layers for this system. Fig. 3(b), Fig. 4(b), Fig. 5(b), and Fig. 6(b) show the Lyapunov exponent spectra of the system versus $a, b, c$ and $d$, respectively. An interesting result is the same bifurcation diagrams and Lyapunov exponent spectra of this new system with respect to $b$ and $d$, which can not be derived from its three equations.
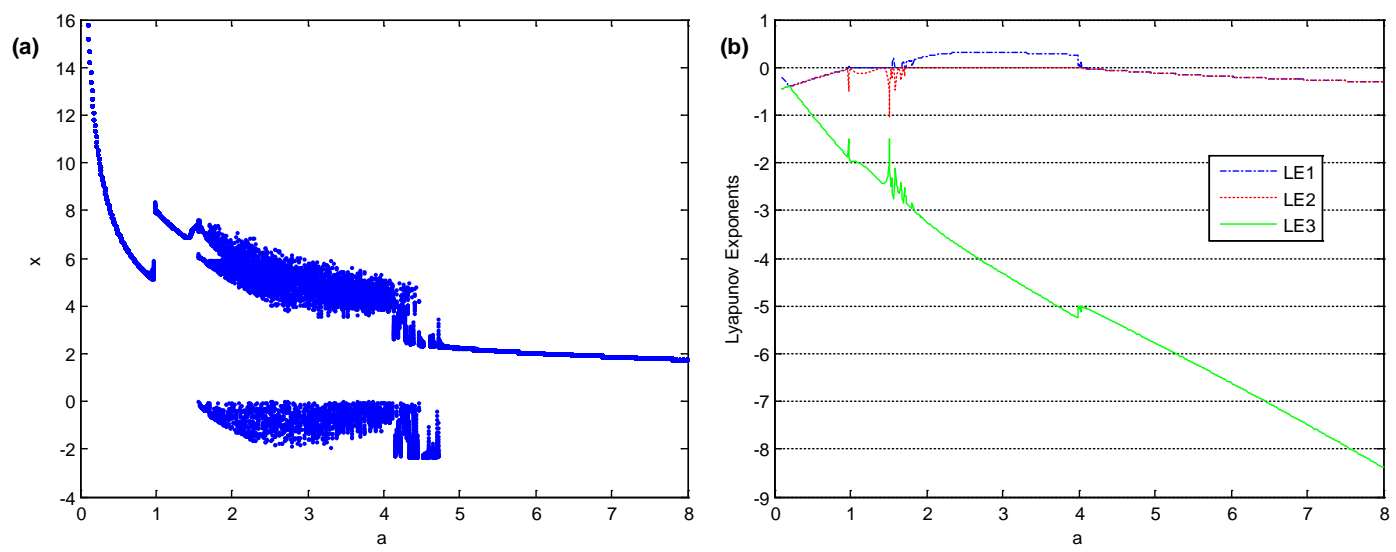

Fig. 3 (a) Bifurcation diagram and (b) Lyapunov exponent spectrum as a function of $a$. Here, $(b, c, d)=(5,0.5,1)$ and initial states $(x(0), y(0), z(0))=(0.1,0.1,0.1)$. 
(a)

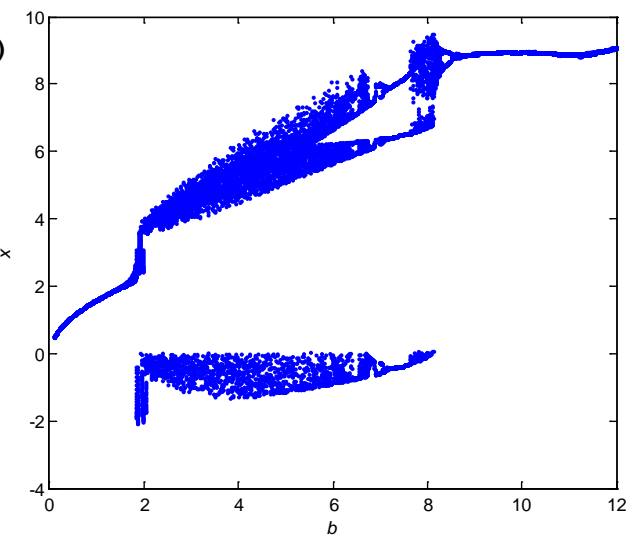

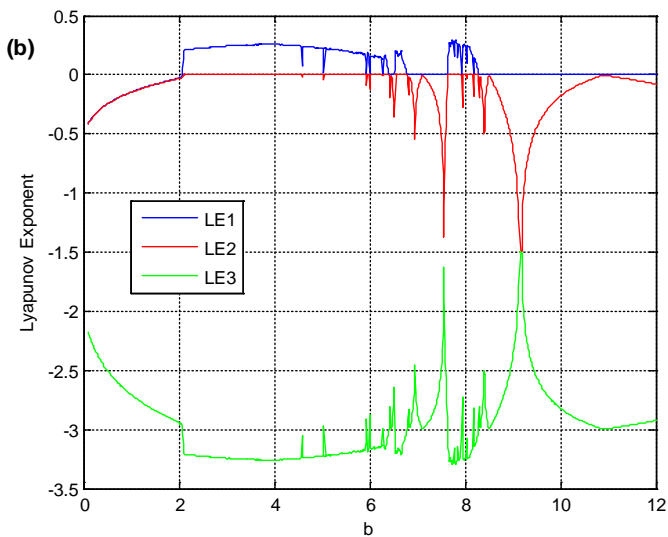

Fig. 4 (a) Bifurcation diagram and (b) Lyapunov exponent spectrum as a function of $b$. Here, $(a, c, d)=(2,0.5,1)$ and initial states $(x(0), y(0), z(0))=(0.1,0.1,0.1)$.
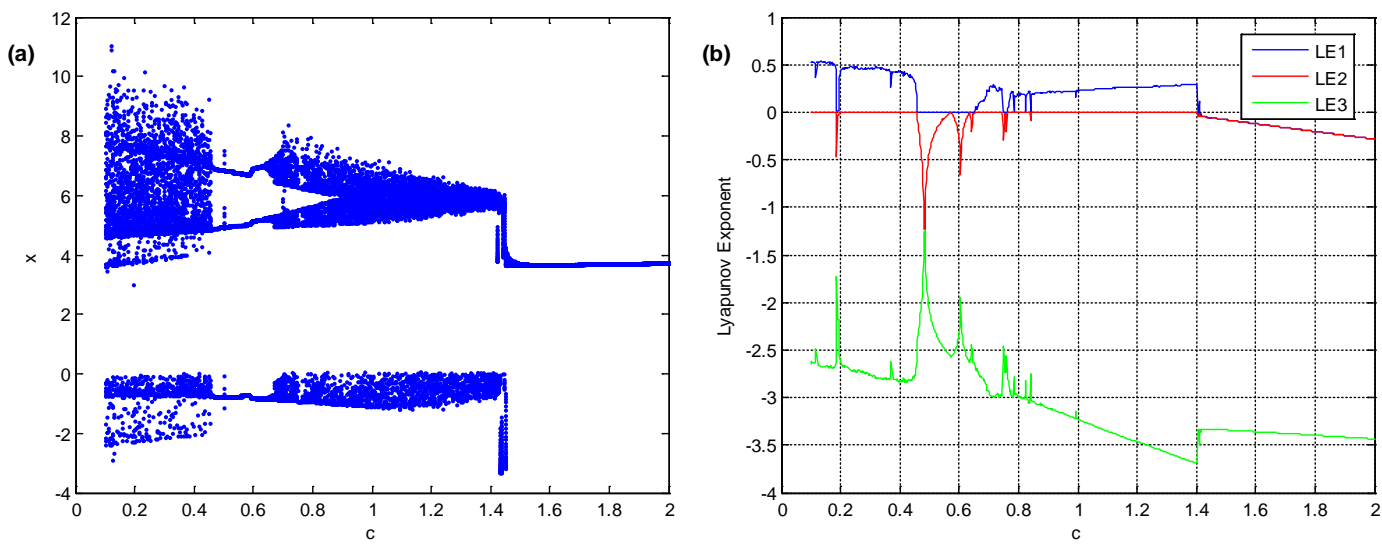

Fig. 5 (a) Bifurcation diagram and (b) Lyapunov exponent spectrum as a function of $c$. Here, $(a, b, d)=(2,5,1)$ and initial states $(x(0), y(0), z(0))=(0.1,0.1,0.1)$.
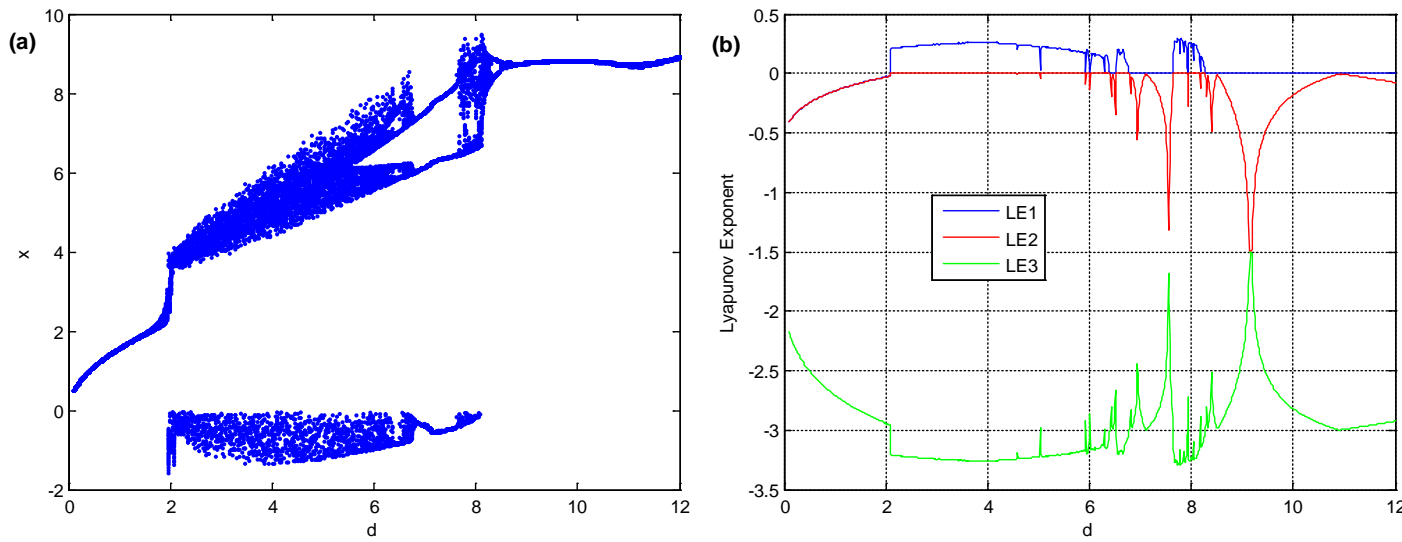

Fig. 6 (a) Bifurcation diagram and (b) Lyapunov exponent spectrum as a function of $d$. Here, $(a, b, c)=(2,5,0.5)$ and initial states $(x(0), y(0), z(0))=(0.1,0.1,0.1)$.

\section{Conclusions}

In summary, by means of the addition of nonlinear terms into the second and third equations of three-dimensional system, a new butterfly-shaped chaotic system with six terms is proposed. And its phase diagram and these Poincaré maps with three conditions are then given. Four mainly dynamical properties: symmetry, equilibrium points, Lyapunov exponent spectra, and bifurcation diagrams are discussed. Numerical results show that the attractor of the system has of fraction dimension. Since the dynamical properties and bifurcation diagrams are different from the previous chaotic systems, this 
new chaotic system will provide a new meaningful template for developing the useful chaos and controlling chaos.

\section{Acknowledgements}

This research was supported by the Research Foundation of Education Bureau of Zhejiang Province of China under Grant No. Y201223107 and the Research Foundation of Science School of Hangzhou Dianzi University under Grant No. KYXM201308.

\section{References}

[1] E.N. Lorenz: J. Atomos. Sci., Vol. 20(1963), p. 130

[2] O.E. Rössler: Phys. Lett. A, Vol. 57(1976), p. 397

[3] Genesio and A. Tesi: Automatica, Vol. 28(1992), p. 531

[4] G.R. Chen and T. Ueta: Chaos, Vol. 9(1999), p. 1465

[5] J.H. Lü and G.R. Chen: Int. J. bifurc. Chaos, Vol. 12(2002), p. 659

[6] E. Ott, C. Grebogi and J.A. Yorke: Phys. Rev. Lett., Vol. 64(1990), p. 1196

[7] K. Pyragas: Phys. Lett. A, Vol. 70(6)(1992), p. 421

[8] S. Boccaletti, C. Grebogi, Y.C. Lai, H. Mancini and D. Maza: Phys. Rep., Vol. 329(2000), p. 103

[9] W. Klonowski: Chaos Solitons Fractals, Vol. 14(2002), p. 1379

[10]H. Yang and G.P. Jiang: IEEE Trans. Circuits Syst. II Express Briefs, Vol. 60(2013), p. 232

[11]C.K. Volos, I.M. Kyprianidis and I.N. Stouboulos: Signal Process, Vol. 93(2013), p. 1328

[12]X.Y. Li and Q.J. Ou: Nonlinear Dyn., Vol. 65(2011), p. 255 